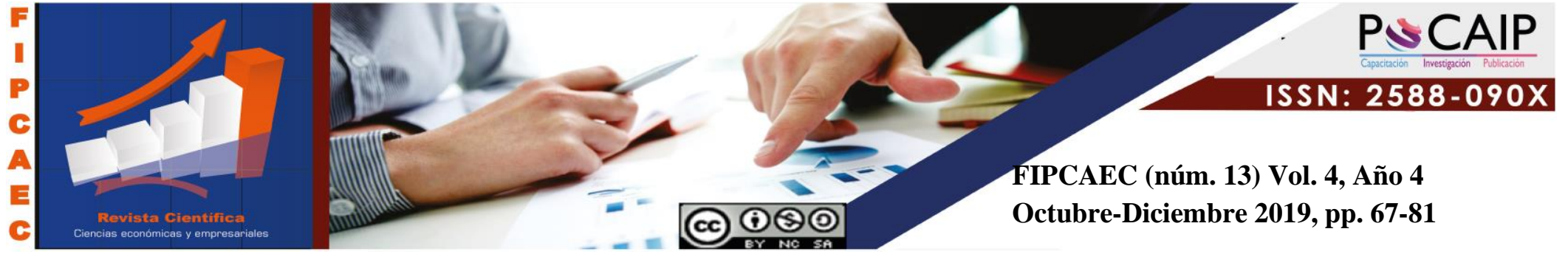

DOI: DOI 10.23857/fipcaec.v4i12.87

\title{
Aprendizaje organizacional y la gestión de calidad en organizaciones cooperativa
}

Organizational learning and quality management in cooperative organizations

Aprendizagem organizacional e gestão da qualidade em organizações cooperativas

\author{
Stalin Carmelo Mendoza-Orellana ${ }^{1}$ \\ stalincmo@yahoo.es \\ https://orcid.org/0000-0003-4789-4139
}

Correspondencia: stalincmo@yahoo.es

* Recepción: 28/ 07/ 2019 *Aceptación: 20/09/ 2019 *Publicación: 15 /10/ 2019

1 Especialista en Diseño Curricular por Competencias, Magíster en Gerencia Educativa Profesional, Ingeniero Industrial, Decano de la Universidad Laica Eloy Alfaro de Manabí, Facultad de Ingeniería Industrial, Manta, Ecuador. 


\title{
Resumen
}

La investigación es de tipo correlacional - transeccional, con el objetivo de determinar la relación entre el aprendizaje organizacional y la gestión de calidad de la cooperativa de suministros tecnológicos Lormart, ubicada en la ciudad de Manta - Ecuador. La población estuvo conformada por 23 personas de la empresa de estudio. Los resultados de ,062 se focaliza en una correlación positiva moderada y significación bilateral de ,777 lo cual implica que la tendencia es acercarse a 1, lo cual implica que existe correlación significativa entre las variables y que la gestión de calidad depende proporcionalmente del aumento del aprendizaje organizacional para mejorar y perfeccionarse en beneficio de la organización. La cooperativa de suministros tecnológicos Lormart, se encuentra en capacidad de contar con líderes gerenciales en razón de potenciar las habilidades y competencias del talento humano de la organización.

Palabras clave: Círculo de calidad; cambio organizacional; modernización; comportamiento organizacional.

\begin{abstract}
The research is correlational - transectional, with the objective of determining the relationship between organizational learning and quality management of the Lormart technology supplies cooperative, located in the city of Manta - Ecuador. The population was made up of 23 people from the study company. The results of, 062 focus on a moderate positive correlation and bilateral significance of, 777 which implies that the tendency is to approach 1, which implies that there is a significant correlation between the variables and that quality management depends proportionally on the increase of the organizational learning to improve and improve for the benefit of the organization. The Lormart technology supplies cooperative is able to count on management leaders because of the skills and competencies of the organization's human talent.
\end{abstract}

Keywords: Quality circle; organizational change; modernization; organizational behavior.

\section{Resumo}

A pesquisa é correlacional - transecional, com o objetivo de determinar a relação entre aprendizado organizacional e gestão da qualidade da cooperativa de suprimentos tecnológicos 
Lormart, localizada na cidade de Manta - Equador. A população era composta por 23 pessoas da empresa do estudo. Os resultados de 062 enfocam uma correlação positiva moderada e significância bilateral de 777, o que implica que a tendência é se aproximar de 1 , o que implica que existe uma correlação significativa entre as variáveis e que o gerenciamento da qualidade depende proporcionalmente do aumento da aprendizado organizacional para melhorar e melhorar em benefício da organização. A cooperativa de suprimentos de tecnologia Lormart é capaz de contar com os líderes de gerenciamento por causa das habilidades e competências do talento humano da organização.

Palavras-chave: Círculo de qualidade; mudança organizacional; modernização; comportamento organizacional.

\section{Introducción}

La nueva economía, la globalización y las nuevas tecnologías son algunos de los elementos que han hecho que la gestión del conocimiento vaya adquiriendo cada vez más importancia. El conocimiento, tal como se entiende hoy día, es un recurso que no tan sólo nos permite interpretar nuestro entorno, sino que nos da la posibilidad de actuar. Es un recurso que se halla en las personas $\mathrm{y}$ en los objetos físicos o no que estas personas utilizan, pero también en las organizaciones a las que pertenecen, en los procesos y en los contextos de dichas organizaciones.

En este sentido, la gestión del conocimiento consiste en optimizar la utilización de este recurso mediante la creación de las condiciones necesarias para que los flujos de conocimiento circulen mejor. Lo que se gestiona en realidad, pues, no es el conocimiento en sí mismo, sino las condiciones, el entorno y todo lo que hace posible y fomenta dos procesos fundamentales: la creación y la transmisión de conocimiento. Son diversos los instrumentos que permiten fomentar y mejorar estos dos procesos, pero para que un proyecto de gestión del conocimiento tenga éxito es fundamental observar, interpretar y entender el funcionamiento de las organizaciones.

De acuerdo con lo expuesto en toda organización la principal actividad que marca una diferencia en el grado en que las instituciones les sirven a las personas, es el nivel de respuesta que estas responden a sus necesidades en función de los servicios que ellas prestan. En este orden de ideas, 
el éxito que puede tener la organización al alcanzar sus objetivos y también al satisfacer sus obligaciones sociales depende, en gran medida, del desempeño gerencial de la organización.

En este contexto, en los últimos tiempos se ha hablado acerca del impacto de la era de la información en las organizaciones. Los funcionarios, ejecutivos y trabajadores se enfrentan con regularidad a abrumadoras cantidades de información, es por ello que se deben generar políticas para la formación permanente del personal que forma parte de una organización para que sean más eficaces y eficientes en el procesamiento de la información que a diario reciben.

Debido a que, es a través de la información como se obtiene el aprendizaje, por medio de la selección y utilización aquella (la información) para fomentar la creatividad. La organización que no promueve el aprendizaje, en especial el aprendizaje rápido no puede esperar ser capaz de competir con éxito. La única forma de conservar la ventaja competitiva es asegurarse de que la organización aprenda con mayor rapidez que la competencia.

Para Senge (2011) el aprendizaje organizacional es una cuestión que debe ser abordada desde una perspectiva sistémica, ya que esta permite a la organización una comprensión global de sí misma, las relaciones entre sus diversas áreas y cómo mejor enfocar sus energías a partir de esta comprensión. Parafraseando al autor, se puede definir el aprendizaje organizacional como el proceso a través del cual las personas y las organizaciones incrementan su capacidad para producir y generar los resultados que quieren generar.

Autores como Argyris (1996) afirman que el aprendizaje organizacional ocurre cuando los agentes producen ajustes entre los resultados y las intenciones o consecuencias que se pretenden. Si se descubre una consecuencia, que no es la deseada o pretendida, y el sistema realiza un cambio para ajustar esta divergencia, entonces, se puede decir que hay aprendizaje. Si esto permite el logro consistente de la consecuencia deseada, entonces, habrá efectividad; la cual, es la única razón de su existencia y el aprendizaje la forma de conquistarlo.

Se puede decir entonces, que el aprendizaje es una herramienta que debe emplear la organización para hacer que las personas que la conforman realmente se sientan comprometidas con el logró de las metas y objetivos de la misma. Una organización que se preocupa por el aprendizaje de su 
personal es una organización que tiene garantizado parte del éxito en el competitivo mercado.

De igual manera para Gore (1998), el aprendizaje organizacional no es una técnica, sino que surge de una actitud, una predisposición para analizar críticamente las acciones y darles sentido. Aprender a usar el potencial educativo de las organizaciones es buscar vías para darle sentido a la experiencia y entender mejor las demandas del entorno para responder a ellas, evitar la formación improductiva y las frustraciones a las que inevitablemente llevan los enfoques tradicionalistas y asumir la responsabilidad junto con el auto perfeccionamiento y el autodesarrollo.

Desde esta concepción, el aprendizaje no solo representa la adquisición de nueva información y habilidades sino, fundamentalmente, una actividad social que se expresa en las organizaciones que permite recoger e integrar distintas experiencias, conocimientos, habilidades y destrezas en torno a una comunidad, donde unos aprenden de otros y todos trabajan por un fin común. La clave es entender el aprendizaje como inseparable del trabajo cotidiano donde se abren espacios para abordar los problemas, aclarar diferencias y fomentar una concepción de sentimiento mutuo con la organización; implica canalizar las acciones individuales, grupales y organizacionales en ambientes de trabajo flexibles, adaptables y exitosos, elevando la calidad del trabajo de las personas y equipos, manteniendo un proceso continuo de desarrollo organizacional.

Aramburu-Goya (2000) aborda dos enfoques sobre el aprendizaje organizacional, el cambio y el conocimiento. Según el primero, las organizaciones que aprenden son aquellas que buscan ampliar su capacidad de adaptarse al cambio continuo en forma ofensiva y sistemática. En el segundo, el aprendizaje organizacional constituye el desarrollo de habilidades y estrategias que permiten que la organización use el conocimiento para lograr sus metas. En concordancia con lo expuesto, se puede decir que una organización que no esté ganada a la idea de aprender y de que las personas que forman parte de ellas se actualicen y mejoren a nivel profesional y emocional, son organizaciones condenadas al fracaso.

Huber (citado en Argyris, 2001) relaciona el aprendizaje organizacional con la capacidad de adquirir información y hacer que los agentes tengan acceso a ella. En tanto que Schein (citado en Argyris, 2001) relaciona el aprendizaje con la habilidad de fomentar una cultura capaz de 
apreciar los supuestos básicos compartidos por el grupo, revelar la disfuncionalidad y suscitar su transformación. En este sentido, Chirinos Martínez, Perozo Martín \& Rodríguez Acasio (2019), indican que las organizaciones requieren de gerentes dirijan efectivamente la organización en medio de una sociedad cambiante, dinámica, competitiva, por lo cual se requiere generar la formación permanente sobre el aprendizaje organizacional con la finalidad de promover la generación de posibilidades en alcanzar niveles de calidad en su gestión.

Por tal motivo la actual investigación tiene por objetivo determinar la relación entre el aprendizaje organizacional y la gestión de calidad de la cooperativa de suministros tecnológicos Lormart, ubicada en la ciudad de Manta - Ecuador.

\section{Desarrollo}

\section{Aprendizaje organizacional}

Para Peter Senge (2005, p. 256), el aprendizaje organizacional radica en la comprensión global de la organización y de las relaciones entre sus partes componentes; enmarca su concepción en una perspectiva sistémica. Senge (2005) entiende el aprendizaje de un modo peculiar:

El verdadero aprendizaje llega al corazón de lo que significa ser humano, a través del aprendizaje nos recreamos a nosotros mismos, a través del aprendizaje nos capacitamos para hacer algo que antes no podíamos, a través del aprendizaje percibimos nuevamente el mundo y nuestra relación con él, a través del aprendizaje ampliamos nuestra capacidad para crear, para formar parte del proceso generativo de la vida. (p. 256)

Esta visión del aprendizaje organizacional, implica la creación de capacidades internas en la organización que le permite adaptarse al entorno y enfrentar tanto la situación actual como el futuro, logra además obtener una ventaja competitiva que reúne las características necesarias para garantizar la sustentabilidad en el tiempo. El aprender es un proceso lento que requiere de un período de desarrollo largo y costoso y que se sustenta en el hombre como principal eje de la organización; esta capacidad a su vez, se convierte en un factor clave de éxito para el desempeño de la organización. Senge refiere en su teoría cinco disciplinas que son:

- El Dominio Personal referente a aprender a cultivar las tensiones entre la visión y la realidad actual para alcanzar mayores resultados. 


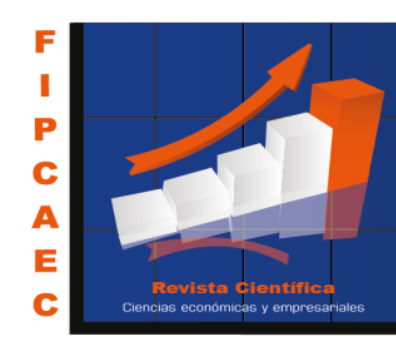

- Los Modelos Mentales que es una disciplina de reflexión e indagación que desarrolla conciencia de las actitudes.

- La Visión Compartida que establece un foco de propósito en común.

- El Aprendizaje en Equipo, que es una disciplina de interacción de grupos a través del diálogo y hábil discusión para aprender a movilizar acciones para alcanzar metas comunes.

- El Pensamiento Sistémico, donde se aprende a dominar mejor la interdependencia y el cambio para entender las fuerzas que norman las consecuencias de nuestros actos.

Dentro de ese contexto Senge (2005, p. 179) refiere que "las organizaciones sólo aprenden a través de individuos que aprenden" y que "el aprendizaje individual no garantiza el aprendizaje organizacional, pero no hay aprendizaje organizacional sin el aprendizaje individual". Por ello, debe asegurarse que el aprendizaje llegue al resto de la organización fomentando la comunicación e integración de las personas, viendo el cambio como actitud permanente, para manejar la complejidad, aprendiendo de los errores y alentando las discusiones abiertas y colaboración entre empleados.

Igualmente, debe hacerse énfasis en invertir en un verdadero programa de aprendizaje y no sólo en capacitación, donde no se relaciona lo recibido con el mejoramiento de la práctica y meses después es poco lo que se recuerda del adiestramiento y menos aún de la contribución del mismo al desempeño. Esto debido a que durante un proceso de capacitación se enfatiza en los contenidos, la pregunta es que se enseña y la actitud es de recibir, mientras que en un proceso de aprendizaje se enfatiza en los procesos, la pregunta es qué se cambia y la actitud es de aplicar.

En concordancia con lo expuesto, el aprendizaje no solo representa la adquisición de nueva información y habilidades sino, fundamentalmente, una actividad social que se expresa en las organizaciones que permite recoger e integrar distintas experiencias, conocimientos, habilidades y destrezas en torno a una comunidad, donde unos aprenden de otros y todos trabajan por un fin común.

La clave es entender el aprendizaje como inseparable del trabajo cotidiano donde se abren 
espacios para abordar los problemas, aclarar diferencias y fomentar una concepción de sentimiento mutuo con la organización; implica canalizar las acciones individuales, grupales y organizacionales en ambientes de trabajo flexibles, adaptables y exitosos, elevando la calidad del trabajo de las personas y equipos, manteniendo un proceso continuo de desarrollo organizacional.

\section{Disciplinas del aprendizaje organizacional desde la perspectiva de Senge}

Dominio Personal: Referido al manejo de la tensión creativa a través de clarificar la visión y enfrentar la realidad. Conocido como la expresión que se usa para la disciplina del crecimiento y el aprendizaje personal. La gente con alto nivel de dominio personal expande continuamente su aptitud para crear los resultados que busca en la vida. Esa búsqueda de aprendizaje continuo es la que permite que surja el espíritu de la organización inteligente, que trasciende más allá de la competencia y las habilidades e implica abordar los retos de una manera creativa y no meramente reactiva.

Aprendizaje en Equipo: Cuyos elementos de estudio corresponden al manejo del diálogo, la discusión y las rutinas defensivas, basados en que las personas además de conocimientos poseen sentimientos y emociones, y que debe hacerse de la organización un lugar donde las mismas se encuentren y trabajen gustosamente, además de fomentar la confianza, las conversaciones y el afecto entre las personas.

El ambiente emocional de la empresa se relaciona con el lenguaje y la forma de comunicación, pues revela claramente cómo es el inconsciente colectivo de la organización, que estilo de liderazgo opera y la manera en que se manejan las relaciones interpersonales. El idioma que propicia el aprendizaje y la aplicación del conocimiento tiene características que vale la pena resaltar, pues las palabras como tal contienen energía y mensajes que pueden producir estados anímicos positivos o negativos, entre estos se encuentran:

El principal problema en un área de trabajo es el miedo. Si la comunicación usa términos de amenazas, desconfianza o inseguridad, neurofisiológica mente se limita gravemente la capacidad de trabajo y aprendizaje de la persona. En este orden de ideas Senge (2000) plantea algunas estrategias para desarrollar el aprendizaje en equipo: 
considera que esta actividad auxiliar a los miembros de la organización a desempeñar su trabajo actual, sus beneficios pueden prolongarse a toda su vida laboral y pueden contribuir al desarrollo, por otra parte ayudan al individuo en el manejo de responsabilidades futuras, independientemente a las actuales.

El contenido de la capacitación puede involucrar cuatro tipos de cambio de comportamiento: a.) transmisión de información como elemento esencial para la toma de decisiones gerencial, b.) desarrollo de habilidades sobre todo de aquellas destrezas y conocimientos directamente relacionados con el desempeño del cargo actual o de posibles ocupaciones futuras, c.) Desarrollo o modificaciones de actitudes relacionadas al aumento de la motivación, desarrollo de la sensibilidad del personal de gerencia y supervisión, adquisición de nuevos hábitos y actitudes entre otros, d.) desarrollo de conceptos que permita facilitar la aplicación de conceptos en la práctica administrativa o para elevar el nivel de generalización pensando en términos globales y amplios.

Los beneficios de la capacitación se orientan a la organización como tal, al individuo y repercute en las relaciones humanas, internas y externas. En relación al beneficio organizacional permite lograr mayor rentabilidad y actitudes más positivas, mejora el conocimiento del puesto a todos los niveles, eleva la moral de la fuerza de trabajo, ayuda al trabajador a identificarse con los objetivos de la organización, fomenta la autenticidad, la apertura y la confianza, agiliza la toma de decisiones y la solución de problemas, promueve la comunicación en toda la organización e incrementa la productividad y la calidad del trabajo entre otros.

Por otro lado, el beneficio para el individuo se orienta a contribuir positivamente al manejo de conflictos y tensiones, lo ayuda para la toma de decisiones y solución de problemas, incrementa el nivel de satisfacción con el puesto, permite el logro de metas individuales, desarrolla un sentido de progreso en muchos campos y elimina temores a la incompetencia o la ignorancia individual. 


\section{Fases para alcanzar el aprendizaje organizacional}

1era Fase: Generación de Información: Consiste en hacer uso de los recursos disponibles para crear una infraestructura y crear conocimiento e ir creando la memoria organizacional. No solo es importante poner a disposición la información sino dar la capacitación necesaria para entender el contexto de esa información y estimularlos para que compartan sus perspectivas con los demás.

2da Parte: Integración y Diseminación: Todos deben entender el propósito de la organización y las relaciones de cada tarea con todo. Compartir la información que cada uno va generando puede ser la diferencia de incurrir en nuevos errores o no. Deben difundirse los encuentros grupales de discusión abierta con respecto a las situaciones que se presentan en lugar de evadirlos, pero estos deben formar parte de la cultura organizacional, no que ocurra una sola vez o como algo especial.

3era Parte: Interpretación colectiva: Este paso puede darse entre dos personas o entre toda la unidad. Representa un tiempo de diálogo y de participación igualitaria. Los participantes aportan los datos que han generado y las conclusiones a que han llegado por su propia experiencia, no informes de otras personas. Cuando se les da participación a todos, se puede actuar de manera natural y llegar a decisiones mucho más acertadas. Esta fase se refiere a convertir la interpretación colectiva en acción local, es decir, descentralizar la toma de decisiones para aumentar la flexibilidad y rapidez.

4ta Parte: Autoridad para asumir responsabilidad de actuar: La organización no puede aprender si los individuos o grupos actúan únicamente basados en sus propias conclusiones, sin estar informados de los acontecimientos y perspectivas de otros. Por eso para que los miembros de la organización puedan actuar deben:

- Disponer de autonomía para efectuar cambios cuando los necesiten.

- Los que actúen deben captar, observar y reflexionar sobre los resultados.

- Se debe tener una estructura para medir los resultados y captar lecciones aprendidas, lo cual lleva a empezar nuevamente el ciclo.

- Un proyecto no se considera completo hasta que no haya formulado lecciones aprendidas, los puntos de acción para el futuro. 
Las organizaciones mediante el aprendizaje organizacional pueden redescubrirse en pro de generar planes estratégicos que le permitan crecer permanentemente desde la concepción sistémica de abordaje de la realidad, contando así con líderes gerenciales en pro de promover acciones favorables para la articulación de una organización inteligente.

\section{Metodología de la investigación}

La investigación es de tipo correlacional - transeccional, de ese modo, se procuró determinar la relación entre el aprendizaje organizacional y la gestión de calidad de la cooperativa de suministros tecnológicos Lormart, ubicada en la ciudad de Manta - Ecuador, para lo cual se sustentó en los planteamientos de Hernández, Fernández, Baptista (2014).

La población estuvo conformada por 23 personas que laboran en la cooperativa de suministros tecnológicos Lormart, a quienes se les aplicó un programa de formación en aprendizaje organizacional para luego medir su incidencia en la gestión de calidad de la empresa, esto mediante el grado correlación establecido desde el coeficiente de Pearson, obteniéndose la información por medio de la aplicación de un instrumento en escalamiento tipo Likert de 76 ítems, con un coeficiente de alfa de Cronbach de 0,92 catalogándose como altamente confiable.

\section{Resultados}

\section{Cuadro 1}

Aprendizaje organizacional y gestión de calidad

\begin{tabular}{llr|r} 
& & Ap & \multicolumn{1}{c}{ Gc } \\
\hline \multirow{2}{*}{ Ap } & Correlación de Pearson & 1 &, 062 \\
\cline { 2 - 4 } & Sig. (bilateral) & &, 777 \\
\cline { 2 - 4 } & N & 23 & 23 \\
\hline \multirow{3}{*}{ Gc } & Correlación de Pearson &, 062 & 1 \\
\cline { 2 - 4 } & Sig. (bilateral) &, 777 & \\
\hline & $N$ & 23 & 23 \\
\hline
\end{tabular}


Los resultados de ,062 se focaliza en una correlación positiva moderada y significación bilateral de ,777 lo cual implica que la tendencia es acercarse a 1, lo cual implica que existe correlación significativa entre las variables y que la gestión de calidad depende proporcionalmente del aumento del aprendizaje organizacional para mejorar y perfeccionarse en beneficio de la organización.

Por lo tanto, se rechaza la hipótesis nula y se acepta la afirmativa, quedando expresada:

H1: El aprendizaje organizacional tiene relación significativa con la gestión de calidad de la cooperativa de suministros tecnológicos Lormart.

\section{Conclusiones}

La cooperativa de suministros tecnológicos Lormart, se encuentra en capacidad de contar con líderes gerenciales en razón de potenciar las habilidades y competencias del talento humano de la organización, comprendiendo que este es el principal factor a impulsar, siendo concordante con lo expuesto por Aldana, Salón \& Guzmán (2019), de ese modo, la población de estudio posee conocimientos sobre como diseñar y abordar estratégicamente la realidad de la empresa, analizando el contexto global como escenario para tomar decisiones en beneficio de crecer como equipo de trabajo, bajo el enfoque de mejoramiento continuo.

\section{Referencias}

1. Aldana, J., Salón, M., \& Guzmán, N. (2019). Liderazgo sistémico en las competencias gerenciales docentes universitarias. CIENCIAMATRIA, 5(8), 50-74. https://doi.org/10.35381/cm.v5i8.87

2. Argyris, C. y Schön, D. A. (1996) Aprendizaje organizacional: Vol. 2. Teoría y Práctica.Addison-Wesley, Massachussets.

3. Aramburu Goya, N. (2000). Un estudio del aprendizaje organizativo desde la perspectiva del cambio: Implicaciones estratégicas y organizativas. Tesis doctoral presentada ante la Universidad de Deusto. San Sebastián 
4. Chiavenato, I (2007). Administración de recursos humanos. Editorial McGraw-Hill. México.

5. Chirinos Martínez, A., Perozo Martín, R., \& Rodríguez Acasio, F. (2019). Imágenes gerenciales de Organizaciones bajo un entorno versátil y competitivo. Revista Arbitrada Interdisciplinaria Koinonía, 4(8), 4-23. doi:http://dx.doi.org/10.35381/r.k.v4i8.224

6. Gore, E. (1998). Desarrollo de competencias y aprendizaje organizacional. Presentado en el XI Congreso Nacional de Desarrollo y Capacitación, organizado por la Asociación de Desarrollo y Capacitación de la Argentina. Mar del Plata, 26 al 28 de octubre de 1998. Recuperado de http://www.udesa.edu.ar/files/img/Administracion/dtn03.pdf

7. Hernández, R., Fernández, C., \& Baptista, P. (2014). Metodología de la investigación. México, Mc Graw Hill Hispanoamericana. Hill Internacional.

8. Robbins, S. y Coulter, M. (1996). Administración. México: Prentice Hall.

9. Senge, P. (2005). La quinta disciplina. Editorial Granica, Barcelona.

10. Senge, P. (2011) Estrategias para liderar el aprendizaje organizacional. Recuperado de http://www.losandes.com.ar/notas/2011/7/3/peter-senge-estrategias-para-lideraraprendizaje-organizacional-578152.asp

\section{References}

1. Aldana, J., Salón, M., \& Guzmán, N. (2019). Systemic leadership in university teaching management skills. SCIENCE, 5 (8), 50-74. https://doi.org/10.35381/cm.v5i8.87

2. Argyris, C. and Schön, D. A. (1996) Organizational learning: Vol. 2. Theory and Practice. Addison-Wesley, Massachusetts.

3. Aramburu Goya, N. (2000). A study of organizational learning from the perspective of change: Strategic and organizational implications. Doctoral thesis presented to the University of Deusto. Saint Sebastian 
4. Chiavenato, I (2007). Human resources management. McGraw-Hill editorial. Mexico.

5. Chirinos Martínez, A., Perozo Martín, R., \& Rodríguez Acasio, F. (2019). Management images of Organizations under a versatile and competitive environment. Interdisciplinary Arbitrated Review Koinonía, 4 (8), 4-23. doi: http: //dx.doi.org/10.35381/r.k.v4i8.224

6. Gore, E. (1998). Skills development and organizational learning. Presented at the XI National Congress of Development and Training, organized by the Association of Development and Training of Argentina. Mar del Plata, October 26-28, 1998. Retrieved from http://www.udesa.edu.ar/files/img/Administracion/dtn03.pdf

7. Hernández, R., Fernandez, C., \& Baptista, P. (2014). Investigation methodology. Mexico, Mc Graw Hill Hispanic American. Hill International

8. Robbins, S. and Coulter, M. (1996). Administration. Mexico: Prentice Hall.

9. Senge, P. (2005). The fifth discipline. Editorial Granica, Barcelona.

10. Senge, P. (2011) Strategies to lead organizational learning. Recovered from http://www.losandes.com.ar/notas/2011/7/3/peter-senge-estrategias-para-lideraraprendizaje-organizacional-578152.asp 